and that a survival signature in tissue adjacent to the tumor correlated with survival in patients with HCC.

Original article Hoshida Y et al. (2008) Gene expression in fixed tissues and outcome in hepatocellular carcinoma. N Engl J Med 359: 1995-2004

\section{Circulating cancer cells predict relapse in patients with gastric cancer and CRC}

At least one-third of patients with gastric cancer or colorectal cancer (CRC) suffer relapse after radical surgery for early-stage disease; new systems, therefore, are needed to identify patients at high risk of recurrence. Yie et al. previously demonstrated that the presence of circulating cancer cells (CCCs) that express survivin mRNA could predict recurrence and metastasis in patients with breast cancer. In this study, the same authors investigated whether survivin-expressing CCCs had a similar predictive value in patients with gastric cancer or CRC.

The study included 55 patients with gastric cancer, 86 patients with CRC and 87 healthy volunteers (controls). The authors' detection technique was used to measure the amount of survivin mRNA in participants' peripheral blood.

Significant differences in mean amounts of survivin mRNA were observed in CCCs in peripheral blood from patients with early stage and metastatic CRC, and also between patients with $\mathrm{CRC}$ and controls. Significant associations were observed between survivin mRNA concentrations and disease stage, nodal status, and extent of tumor penetration in patients with gastric cancer or CRC. Tumors with an adverse prognosis were associated with high blood levels of survivin mRNA. In patients with gastric cancer, detection of survivin-expressing CCCs was a significant risk factor for metastasis. The rate of recurrence was significantly elevated in patients with survivin-expressing CCCs across the whole cohort.

The measurement of survivin-expressing CCCs could be used to predict and diagnose metastasis and recurrence in patients with gastric and CRC.

Original article Yie S et al. (2008) Detection of survivinexpressing circulating cancer cells (CCCs) in peripheral blood of patients with gastric and colorectal cancer reveals high risks of relapse. Ann Surg Oncol 15: 3073-3082

\section{Best practice in the management of malignant germ-cell tumors of the ovary}

Malignant germ-cell tumors of the ovary are rare and can be treated with cisplatin-based combination chemotherapy or ovariectomy. To achieve high cure rates for this disease a multidisciplinary approach is required. Palenzula et al. carried out a retrospective study to analyze outcomes in a cohort of patients who underwent surgical or medical management for malignant germ-cell tumors of the ovary.

The study included 60 patients treated for primary malignant germ-cell tumors of the ovary at two centers in France, between 1985 and 2003. The patient's case records were obtained and analyzed for information on diagnosis, surgery and medical decisions. A total of 38 tumors were stage I, and 22 were advanced. The mean follow-up period was 5.9 years. During follow-up, $16.7 \%$ of patients experienced relapse. In general, relapse occurred more frequently in patients with localized disease, possibly because those with advanced disease had received adjuvant chemotherapy. Overall survival at 5 years of follow-up was $96.7 \%$ and event-free survival was $79.2 \%$. Patients who underwent postsurgical observation and those with yolk-sac tumor had an increased risk of relapse. The type of surgery or chemotherapy used had no prognostic value.

The authors conclude that outcomes of patients with malignant germ-cell ovarian tumors can be greatly influenced by accurate staging and adherence to surgical and medical guidelines.

Original article Palenzuela G et al. (2008) Comprehensive staging allows for excellent outcome in patients with localized malignant germ cell tumor of the ovary. Ann Surg 248: 836-841 